\title{
Virus-PEDOT Nanowires for Biosensing
}

\author{
Jessica A. Arter, ${ }^{\dagger}$ David K. Taggart, ${ }^{\dagger}$ Theresa M. McIntire, Reginald M. Penner, ${ }^{*}$ and \\ Gregory A. Weiss*
}

Department of Chemistry, University of California, Irvine, California 92697-2025, United States

\begin{abstract}
The separate fields of conducting polymer-based electrochemical sensors and virus-based molecular recognition offer numerous advantages for biosensing. Grafting M13 bacteriophage into an array of poly (3,4-ethylenedioxythiophene) (PEDOT) nanowires generated hybrids of conducting polymers and viruses. The virus incorporation into the polymeric backbone of PEDOT occurs during electropolymerization via lithographically patterned nanowire electrodeposition. The resultant arrays of virus-PEDOT nanowires enable real-time, reagent-free electrochemical biosensing of analytes in physiologically relevant buffers.
\end{abstract}

KEYWORDS Nanowires, phage display, PEDOT, biosensing, conducting polymers

$\mathrm{N}$ anowires offer versatile and unique properties for chemical and biological sensing. The chemical sensitivity of nanowires typically results from their high surface-to-volume ratios. ${ }^{1-3}$ The nanometer-scale, in addition to providing capacity for high-density parallelization, is well suited for biological systems. Interest in nanowire-based sensors derives primarily from the potential for the sensor to be label- and reagent-free; direct electrical sensing with nanowires could ultimately deliver a real-time device with the attributes of small size, low cost, and potential for highthroughput measurements. ${ }^{4,5}$ Conventional nanowire biosensors use semiconducting nanowires, surface-modified with receptors such as antibodies, in a field-effect transistor configuration. The binding of a charged analyte molecule to these receptors induces a conductivity change in the nanowire by Coulombically accumulating or depleting charge carriers. ${ }^{4,6}$ Silicon and indium oxide semiconductor nanowires, for example, have been used to directly sense $\mathrm{pH}$, metal ions, small molecules, proteins, ${ }^{6-10}$ lipoproteins, ${ }^{11}$ and DNA. ${ }^{12,13}$ Nanowires have also been used as the basis for biosensors that detect virus particles. ${ }^{14}$

Conducting polymers, such as polythiophene, polypyrrole, and PEDOT, provide new opportunities for the incorporation of receptors into nanowires for biosensing. ${ }^{10,15-18}$ In contrast to metals and inorganic semiconductors, conducting polymers have a high degree of structural malleability and flexibility; additionally, conducting polymers offer some degree of porosity to potentially allow access to solvent and prospective analyte molecules. Nanoparticles, nanowires, and other submicrometer-scale structures have been synthesized to allow tunable charge-transport and offer a wide range of chemical and physical properties. For measurements in biological systems, the stability to physiological

\footnotetext{
* To whom correspondence should be addressed. E-mail: (G.A.W.) gweiss@uci.edu; (R.M.P.) rmpenner@uci.edu.

† Joint first authors.

Received for review: 07/22/2010

Published on Web: 11/01/2010
}

conditions of conducting polymer nanowires provides high intrinsic biocompatibility. ${ }^{15,19}$ Doped conducting polymers have inherent electrical conductivity resulting from the presence of charge carriers and the mobility of the carriers in a conjugated system. ${ }^{20}$ Conducting polymer-based sensors have been used to detect ammonia, ${ }^{21,22}$ chloroform, ${ }^{23}$ hydrogen, ${ }^{24}$ acetic acid, ${ }^{25}$ and other compounds. ${ }^{26}$

The specificity and selectivity of conducting polymer biosensors, either in thin films or nanowires, can be customized with biomolecules providing molecular recognition. ${ }^{27,28}$ The syntheses of conducting polymers can be made compatible with the integration of biomolecules, which typically require aqueous conditions at moderate temperatures and neutral $\mathrm{pH}$. Approaches to incorporate biomolecules into conducting polymers include attachment to a monomer before polymerization, entrapment during synthesis, or conjugation after synthesis. To date, immobilized recognition elements include metal ions, antibodies, DNA, proteins, and enzymes. ${ }^{10,27-34}$ Viruses, which offer versatile platforms for molecular recognition, ${ }^{35-37}$ have not been previously incorporated into conducting polymer nanowire-based biosensors.

M13, a bacteria-infecting virus or bacteriophage, can recognize essentially any analyte by binding to engineered polypeptides displayed on its surface, which can be altered through manipulation of the phage-packaged DNA. ${ }^{35-37}$ The protein coat of the virus provides densely packed receptors for avidity-based binding to analytes. Thus, the receptors selected from phage-displayed libraries can bind to small molecules, ${ }^{38,39}$ proteins, ${ }^{40-44}$ DNA,${ }^{45}$ and viruses. ${ }^{46}$ Inexpensive, readily produced, and available in large quantities, these viruses infect only their host E. coli bacteria. M13 viruses can also form films patterned by an underlying polymer, or template the synthesis of materials through binding to phage-displayed peptides ${ }^{47,48}$ With proven capabilities for engineered molecular recognition and materials, viruses could provide new approaches to electrical conductivity-based biosensing. 
SCHEME 1. Polymerization of (a) EDOT and (b) EDOT with Virus Incorporation

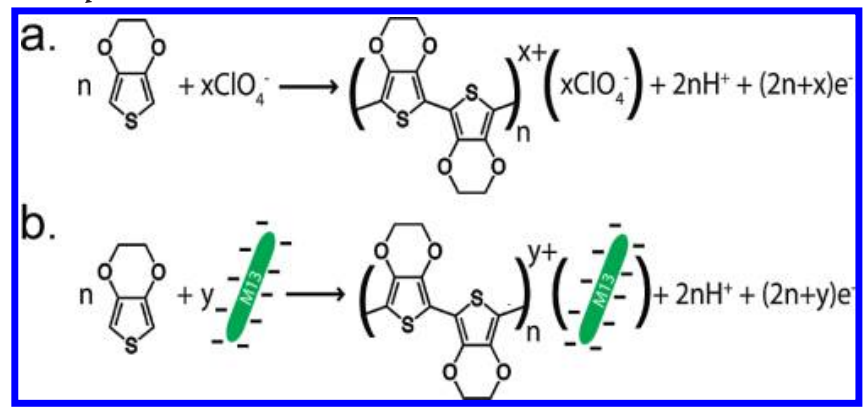

To advance the field, several issues must be addressed. First, can arbitrary patterns of conducting polymer nanowires be fabricated in a controlled, yet high-throughput, manner? Second, how can generalizable molecular recognition motifs be integrated into the nanowires? Third, what versatile molecular recognition scaffolds can tolerate the harsh conditions required for reliable microelectronics fabrication? These challenges require facile synthesis of nanowire structures with entrapped, stable scaffolds for the recognition of analytes. Reported here, we solve these issues through incorporation of M13 viruses into nanowire arrays. Difficulties incorporating viruses into metal nanowires necessitated development of a new lithographically patterned nanowire electrodeposition (LPNE) synthesis using the watersoluble EDOT monomer (Scheme 1a). ${ }^{49,50}$ The approach offers chemical and biological selectivity to the nanowire arrays, without requiring postsynthesis functionalization. The resultant label-free biosensing platform allows direct electrical resistance measurements to detect low concentrations of target analytes.

The LPNE method was used to create devices with linear arrays of several hundred virus-PEDOT hybrid nanowires on glass. This process (Figure 1a) involved the vapor-deposition of a nickel film. After coating the film with a photoresist (Figure 1a-i), photolithographic patterning defined the desired position for the nanowires (Figure 1a-ii). In the key step, oxidative degradation removed the exposed nickel and etched in a side-exposed, nanotrench through the nickel (Figure 1a-iii). The nickel nanotrench was used as the working electrode in a three-electrode cell for electrodeposition of PEDOT nanowires from aqueous 2.5 mM EDOT, $12.5 \mathrm{mM}$ $\mathrm{LiClO}_{4}$, and, for virus-PEDOT hybrid nanowires, $10 \mathrm{nM} \mathrm{M1} 3$ bacteriophage (Figure $1 \mathrm{a}-\mathrm{iv}$ ). The resultant nanowires had a rectangular cross-section with widths dictated by the duration of electrodeposition and heights fixed by the thickness of the nickel layer evaporated during the first step of the process. Efficient removal of the photoresist by acetone treatment, followed by further etching of the remaining nickel, generated an array of freestanding virus-PEDOT nanowires for electrical resistance measurements (Figure 1av). Importantly, the entire LPNE fabrication process was carried out in a conventional chemistry laboratory and did not require a clean room.

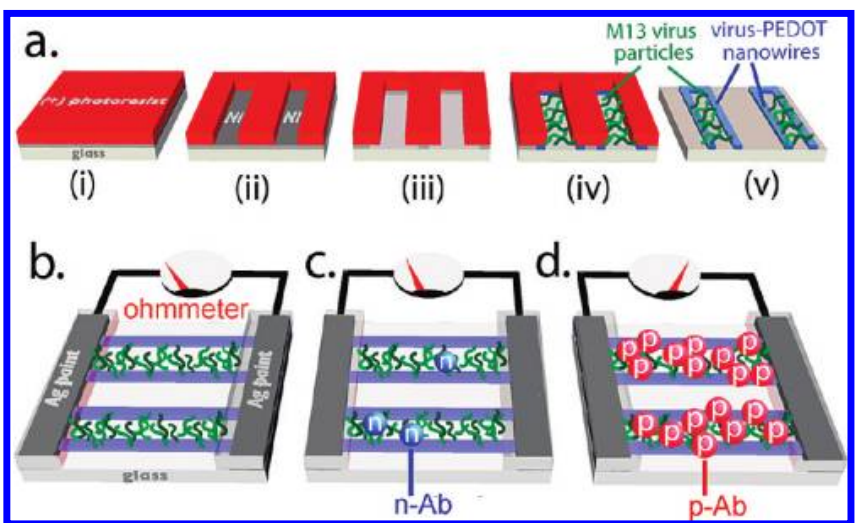

FIGURE 1. (a) Schematic diagram of the LPNE process for the synthesis of virus-PEDOT nanowires. (b) The resultant virus-nanowire device used for resistance-based measurements. (c) Schematic, indicating little to no change in resistance after treatment with a nonbinding negative control antibody ( $\mathrm{n}-\mathrm{Ab}$, blue circles). (d) Depiction of the substantial change in resistance due to the presence of virus-binding positive antibody ( $\mathrm{p}-\mathrm{Ab}$, red circles).

Unlike previous examples of viruses linked covalently or noncovalently to surfaces, the PEDOT synthesis allows for direct encapsulation of viruses into the interiors of nanowires. Synthesis of PEDOT nanowires requires $\mathrm{LiClO}_{4}$ dissolved in a monomeric, aqueous solution of EDOT, and the $\mathrm{ClO}_{4}{ }^{-}$anions are closely associated with the PEDOT nanowires during the oxidative electrodeposition. Our strategy takes advantage of the overall highly negatively charged surface of the phage, a property exploited previously for the coating of phage with cationic polymers. ${ }^{51}$ Thus, the viruses competed with $\mathrm{ClO}_{4}{ }^{-}$ions for electrostatic incorporation into the nanowires (Scheme $1 \mathrm{~b}$ ), and remained stable through multiple steps including drying, washing, and treatment with both acetone and nitric acid $(0.8 \mathrm{M})$.

The electrodeposition of virus-PEDOT nanowires cannot be initiated from a solution containing both EDOT and M13 phage. Instead, a pure PEDOT "primer" was first electrodeposited from a solution containing EDOT (2.5 mM) without the M13 viruses. This PEDOT primer was electropolymerized by twice scanning the potential of the nickel LPNE electrode from $400 \mathrm{mV}$ to $1.025 \mathrm{~V}$ versus a saturated calomel electrode (SCE) at $20 \mathrm{mV} \mathrm{s}^{-1}$. The width of this pure PEDOT primer is estimated to be $20 \mathrm{~nm}$. Next, the deposition solution was exchanged for one containing both EDOT (2.5 $\mathrm{mM})$ and $\mathrm{M} 13$ phage $(10 \mathrm{nM})$. Both of these solutions also contained $12.5 \mathrm{mM} \mathrm{LiClO}_{4}$, which was used to resuspend the phage precipitated during isolation. In the M13-containing EDOT solution, six additional polymerization scans were carried out during which M13 incorporation into the nanowire occurred (Supporting Information, Figure S1a).

Since PEDOT nanowires have not previously been prepared by LPNE, we first characterized the chemical composition of arrays of pure PEDOT nanowires by FTIR and EDX. A comparison between a clean glass surface and an array of PEDOT nanowires supported on glass showed additional carbon content for the PEDOT nanowires as well as a 


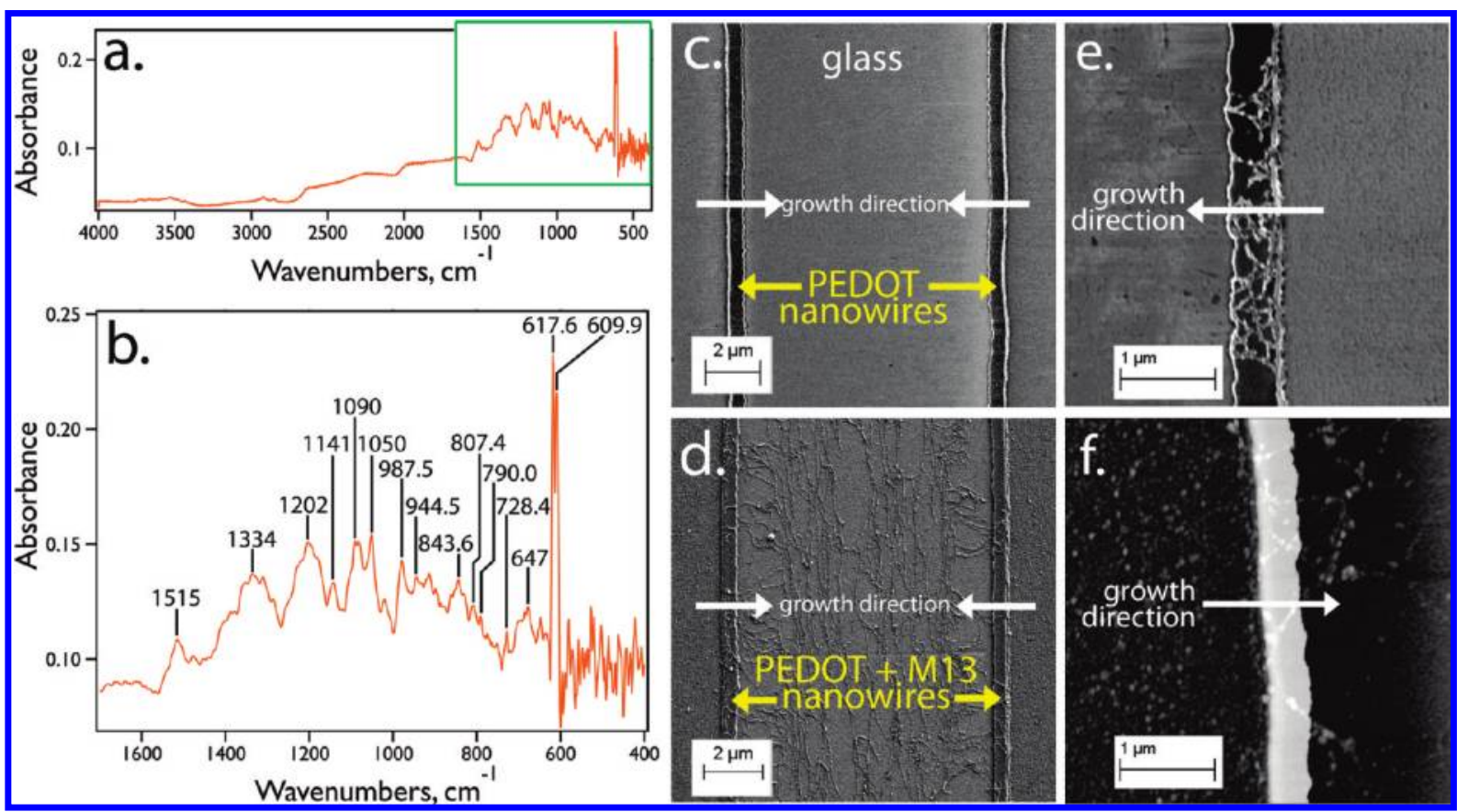

FIGURE 2. (a) FTIR of the PEDOT nanowire array. (b) Inset showing the indicated range of FTIR data from panel a. (c) A secondary electron SEM image of PEDOT nanowires. (d) A secondary electron SEM image of virus-PEDOT wires, where M13 bacteriophage are observed as stringlike particles within and between the nanowires. (e) An in-lens secondary electron SEM image of a single virus-PEDOT wire, where viruses appear as conducting, fibrous particles within the nanowire. (f) An AFM image of a single virus-PEDOT wire.

significant sulfur signal, as expected for this compound (Supporting Information, Figure S2). The transmission FTIR spectra of arrays of pure PEDOT nanowires produced 17 peaks assignable to PEDOT including strong stretches at 1515 and $1334 \mathrm{~cm}^{-1}$ assigned to a $\mathrm{C}=\mathrm{C}$ stretch and peaks at $1202,1141,1090$, and $1050 \mathrm{~cm}^{-1}$ that are assigned to $v(\mathrm{C}-\mathrm{O}-\mathrm{C})$ stretches (Figure 2a,b). Additional spectral assignments are reported in Supporting Information, Table S1. FTIR spectra of virus-PEDOT nanowires showed no additional spectral peaks assignable to the incorporated M13. Taken together, these data demonstrate that nanowires of PEDOT can be prepared by LPNE.

Several lines of evidence from SEM, AFM, fluorescence microscopy, and electrochemical biosensing support the incorporation of viruses into the conducting PEDOT nanowire arrays. First, clear differences between virus and nonvirus PEDOT nanowires are evident in the SEM. Notably, the visualization of such viruses by this technique without sputter coating of a conductive material suggests the integration of the viruses into the conducting PEDOT nanowires. Second, fibrous structures with the dimensions of bundled filamentous viruses are clearly visible (Figure 2d,e). M13 viruses have the dimensions of approximately $1 \mu \mathrm{m}$ in length by $7 \mathrm{~nm}$ in diameter ${ }^{52}$ and can be observed as aggregates within the wires. By comparison, the height of the virus-PEDOT wires was $\sim 60 \mathrm{~nm}$, and the width ranged from 400 to $500 \mathrm{~nm}$. The negative control nanowires synthesized in the absence of viruses do not have such structures (Figure 2c). The small bundles of viruses can also be observed in AFM images and appear quite distinct from the nonvirus containing wires (Figure 2f).

Further characterization by fluorescence microscopy and biosensing both confirmed the successful incorporation of viruses into the PEDOT and demonstrated that the viruses remained intact and fully functional for binding to analytes. A fluorescence assay applying an M13-specific antibody conjugated to fluorescein, followed by thorough wash steps, resulted in selective binding by the antibody to virusfunctionalized nanowires (Figure 3). This fluorescence proved the viruses were sufficiently exposed and functional to allow binding to the antibody. Furthermore, the viruses were very specifically incorporated, and highly localized to the virusPEDOT nanowires, which can be clearly seen from the flat line trace (Figure $3 \mathrm{a}$ ) and the virus-PEDOT line trace (Figure 3b) showing a pattern of periodic fluorescence corresponding to the patterning of the nanowires. In the absence of antibody, no fluorescence is observed (data not shown).

Biosensing with the virus-PEDOT nanowire arrays further demonstrates successful incorporation of fully functional viruses. To fabricate biosensors based upon the nanowire arrays, silver contacts and wires were pasted onto the nanowires exposing the virus-PEDOT nanowires for electrical biosensing measurements; each device exposed 200-300 $\mu \mathrm{m}$ lengths of nanowires and included hundreds of parallel nanowires. The contacts were covered with an insulating paint to prevent interaction with the liquid (Figures 1b, 4a). After thorough drying, the devices were tested for resistance readings between 30 and $400 \mathrm{k} \Omega$, which indicated a suf- 


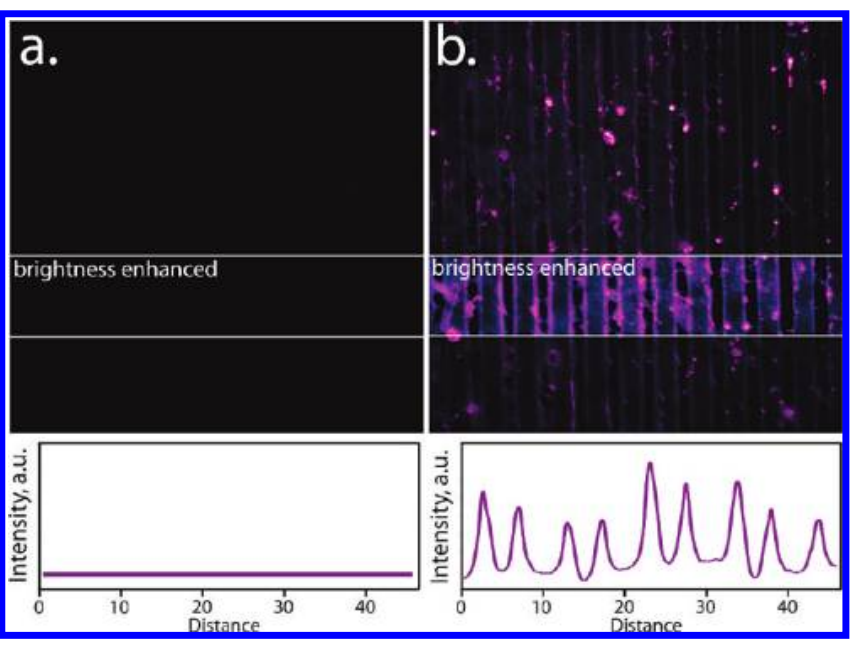

FIGURE 3. (a) A fluorescence microscopy image of PEDOT nanowires after incubation with anti-M13 antibody conjugated to fluorescein. As expected, minimal fluorescence intensity is observed (lower). (b) A fluorescence microscopy image of virus-PEDOT nanowires following incubation with anti-M13 antibody conjugated to fluorescein shows localization of the viruses to the nanowires. Image J analysis quantifies the increased fluorescence intensity at the wires (lower).

ficient number of nanowires in the array for reproducible biosensing. Using an applied bias, $E_{\text {app }}=100 \mathrm{mV}$ across the nanowire array, the current across, I, was measured and converted into resistance, $R=E_{\mathrm{app}} / I$, which was recorded in real-time during immersion in phosphate buffered fluoride solution (PBF). Following attainment of a stable baseline resistance, $15 \mu \mathrm{L}$ aliquots containing known concentrations of the positive antibody ( $\mathrm{p}-\mathrm{Ab})$, capable of binding to M13 viruses, or a negative control antibody (n-Ab), known not to bind the viruses, were pipetted onto the nanowires (Figure $1 \mathrm{c}, \mathrm{d})$. Upon observation of an increase in resistance and a saturated signal, the nanowires were washed thoroughly with PBF. The calibration plot shown in Figure 4c shows the change in resistance from each injection of antibody, $\Delta R$, normalized by the initial electrical resistance measured in pure PBF buffer, $R_{0}$.
Analyte binding to the virus-PEDOT nanowires resulted in a concentration dependent increase in resistance. At the highest concentrations of virus binding antibody (99 nM), an $\sim 40 \%$ increase in the resistance was observed. The lowest concentrations of analyte provide a limit of detection for this array of virus-PEDOT nanowires in the range of 20 $\mathrm{nM}$. As reported here, no aspect of the device has been optimized. Changes to phage-loading, device configuration, nanowire diameter and/or length could improve device sensitivity.

The mechanism of the resistance increase induced by $\mathrm{p}$-Ab binding is incompletely understood, but one possibility is that the binding of $\mathrm{p}-\mathrm{Ab}$ is induced by a charge-gating effect similar to that operating at semiconductor nanowires. Two negative controls support this hypothesis (Figure 4c). First, treatment with PBF in the absence of antibody shows no change in the resistance. Second, the negative antibody (anti-Flag antibody), which again is known not to bind to the viruses, alters the resistance minimally, less than $10 \%$ Furthermore, unmodified PEDOT nanowires, lacking the integrated viruses, changed resistance only minimally, less than $10 \%$, upon treatment with both positive and negative antibodies (Figure 4c). Collectively, these data show that virus-PEDOT hybrid nanowires are capable of directly electrically transducing the specific binding of an antibody to the entrained virus. The results thus demonstrate the viruses integrated into the PEDOT retain functionality, and can alter the properties of the nanowire through molecular recognition of analytes.

Real-time, reagent-free biosensing could provide an essential tool for early disease detection and diagnosis. Toward this goal, the reported virus-PEDOT nanowires provide an effective route for electrical resistance-based sensing in a buffer at physiologically relevant $\mathrm{pH}$, ionicity, and room temperature. The viruses applied here, M13 bacteriophage, are readily amenable to tailoring of their surfaces for molecular recognition using phage display. Thus, the generalizability of M1 3 viruses to recognize a wide range of antigens
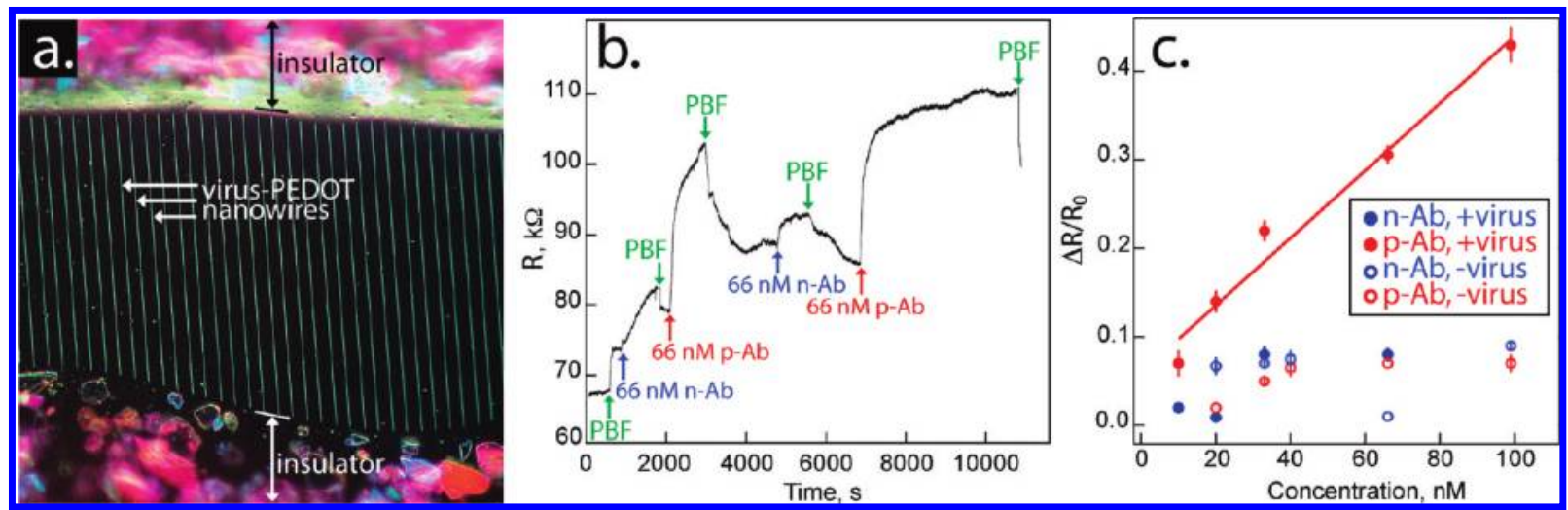

FIGURE 4. (a) Optical micrograph of a virus-PEDOT nanowire array device. (b) Raw biosensing data, taken in real-time with the indicated injections of negative antibody ( $\mathrm{n}-\mathrm{Ab}$, blue arrows), positive antibody ( $\mathrm{p}-\mathrm{Ab}$, red arrows), or washes with PBF buffer (green arrows). (c) $\mathrm{A}$ compilation of all real-time biosensing data, depicted as a calibration curve, in which the resistance change upon injection is plotted versus analyte concentration. 
and disease markers suggests the technique could find broad applicability to clinical diagnostics.

Acknowledgment. The authors thank the Carl Zeiss Center of Excellence for Electron Microscopy for providing access to a SEM and Rob Corn for use of a fluorescence microscope. We thank Keith Donavan and Rosa Pilolli for useful discussions, and Greg Williams for assistance with the SEM and EDX microscopy. T.M.M. thanks Dr. John N. Russell, Jr. at the Naval Research Laboratory for useful discussions. R.M.P. acknowledges support from the National Science Foundation (CHE-0956524) and G.A.W. acknowledges support from the NAID (1 R43 AI074163) and the NCI (R01 CA133592-01) of the National Institutes of Health.

Supporting Information Available. Experimental details, and additional EDX, FTIR, and cyclic voltammetry data are available. This material is available free of charge via the Internet at http://pubs.acs.org

\section{REFERENCES AND NOTES}

(1) Waggoner, P. S.; Craighead, H. G. Lab Chip 2007, 7, 1238-1255

(2) Patolsky, F.; Zheng, G. F.; Lieber, C. M. Anal. Chem. 2006, 78, 4260-4269

(3) Kim, J.; Junkin, M.; Kim, D. H.; Kwon, S.; Shin, Y. S.; Wong, P. K. Gale, B. K. Microfluid. Nanofluid. 2009, 7, 149-167.

(4) Stern, E.; Vacic, A.; Reed, M. A. IEEE Trans. Electron Dev. 2008, $55,3119-3130$

(5) Carlen, E. T.; van den Berg, A. Lab Chip 2007, 7, 19-23.

(6) Cui, Y.; Wei, Q.; Park, H.; Lieber, C. M. Science 2001, 293, 12891292.

(7) Chen, R. J.; Bangsaruntip, S.; Drouvalakis, K. A.; Kam, N. W.; Shim, M.; Li, Y.; Kim, W.; Utz, P. J.; Dai, H. Proc. Natl. Acad. Sci. U.S.A. 2003, 100, 4984-4989.

(8) Wang, W. U.; Chen, C.; Lin, K. H.; Fang, Y.; Lieber, C. M. Proc. Natl. Acad. Sci. U.S.A. 2005, 102, 3208-3212

(9) Li, C.; Curreli, M.; Lin, H.; Lei, B.; Ishikawa, F. N.; Datar, R.; Cote, R. J.; Thompson, M. E.; Zhou, C. W. L. Am. Chem. Soc. 2005, 127 12484-12485.

(10) Ramanathan, K.; Bangar, M. A.; Yun, M.; Chen, W.; Myung, N. V.; Mulchandani, A. I. Am. Chem. Soc. 2005, 127, 496-497.

(11) Tang, T.; Liu, X. L.; Li, C.; Lei, B.; Zhang, D. H.; Rouhanizadeh, M.; Hsiai, T.; Zhou, C. W. Appl. Phvs. Lett. 2005, 86, 103903.

(12) Li, Z.; Chen, Y.; Li, X.; Kamins, T. I.; Nauka, K.; Williams, R. S. Nano Lett. 2004, 4, 245-247.

(13) Hahm, J.; Lieber, C. M. Nano Lett. 2004, 4, 51-54.

(14) Patolsky, F.; Zheng, G. F.; Hayden, O.; Lakadamyali, M.; Zhuang, X. W.; Lieber, C. M. Proc. Natl. Acad. Sci. U.S.A. 2004, 101, $14017-$ 14022

(15) Hangarter, C. M.; Bangar, M.; Mulchandani, A.; Myung, N. V. L. Mater. Chem. 2010, 20, 3131-3140.

(16) Janata, J.; Josowicz, M. Nat. Mater. 2003, 2, 19-24.

(17) Jager, E. W.; Smela, E.; Inganas, O. Science 2000, 290, 15401545.

(18) Xia, L.; Wei, Z.; Wan, M. L. Colloid Interface Sci. 2010, 341, 1-11

(19) Wang, J. ChemPhysChem 2009, 10, 1748-1755
(20) McQuade, D. T.; Pullen, A. E.; Swager, T. M. Chem. Rev. 2000, $100,2537-2574$

(21) Liu, H. Q.; Kameoka, J.; Czaplewski, D. A.; Craighead, H. G. Nano Lett. 2004, 4, 671-675

(22) Chartuprayoon, N.; Hangarter, C. M.; Rheem, Y.; Jung, H.; Myung, N. V. L. Phys. Chem. C 2010, 114, 11103-11108.

(23) Sharma, S.; Nirkhe, C.; Pethkar, S.; Athawale, A. A. Sens. Actuators, B 2002, 85, 131-136.

(24) Virji, S.; Kaner, R. B.; Weiller, B. H. L.Phys. Chem. B 2006, 110 , 22266-22270

(25) Ko, S.; Jang, J. Biomacromolecules 2007, 8, 182-187.

(26) Wang, J.; Chan, S.; Carlson, R. R.; Luo, Y.; Ge, G. L.; Ries, R. S.; Heath, J. R.; Tseng, H. R. Nano Lett. 2004, 4, 1693-1697.

(27) Vidal, J. C.; Garcia-Ruiz, E.; Castillo, J. R. Microchim. Acta 2003, 143, 93-111.

(28) Asplund, M.; von Holst, H.; Inganas, O. Biointerphases 2008, 3, 83-93.

(29) Aravinda, C. L.; Cosnier, S.; Chen, W.; Myung, N. V.; Mulchandani, A. Biosens. Bioelectron. 2009, 24, 1451-1455.

(30) Yoon, H.; Kim, J.-H.; Lee, N.; Kim, B.-G.; Jang, J. ChemBioChem 2008, 9, 634-641.

(31) Tolani, S. B.; Craig, M.; DeLong, R. K.; Ghosh, K.; Wanekaya, A. K. Anal. Bioanal. Chem. 2009, 393, 1225-1231.

(32) Bangar, M. A.; Shirale, D. J.; Chen, W.; Myung, N. V.; Mulchandani, A. Anal. Chem. 2009, 81,2168-2175.

(33) Abasiyanik, M. F.; Senel, M. L. Electroanal. Chem. 2010, 639, 21 26

(34) Hamedi, M.; Herland, A.; Karlsson, R. H.; Inganas, O. Nano Lett. $2008,8,1736-1740$.

(35) Smith, G. P. Science 1985, 228, 1315-1317.

(36) Levin, A. M.; Weiss, G. A. Mol. Biosyst. 2006, 2, 49-57.

(37) Petrenko, V. A.; Smith, G. P. Protein Enq. 2000, 13, 589-592

(38) Goldman, E. R.; Pazirandeh, M. P.; Charles, P. T.; Balighian, E. D.; Anderson, G. P. Anal. Chim. Acta 2002, 457, 13-19.

(39) Rozinov, M. N.; Nolan, G. P. Chem. Biol. 1998, 5, 713-728

(40) Kehoe, J. W.; Kay, B. K. Chem. Rev. 2005, 105, 4056-4072

(41) Kay, B. K.; Hamilton, P. T. Comb. Chem. High Throughput Screening 2001, 4, 535-543.

(42) Scott, J. K.; Smith, G. P. Science 1990, 249, 386-390.

(43) Yang, L. M.; Tam, P. Y.; Murray, B. J.; McIntire, T. M.; Overstreet, C. M.; Weiss, G. A.; Penner, R. M. Anal. Chem. 2006, 78, 32653270 .

(44) Sidhu, S. S.; Koide, S. Curr. Opin. Struct. Biol. 2007, 17, 481-487.

(45) Choo, Y.; Klug, A. Curr. Opin. Biotech. 1995, 6, 431-436.

(46) Gough, K. C.; Cockburn, W.; Whitelam, G. C. L. Virol. Methods 1999, 79, 169-180.

(47) Sanghvi, A. B.; Miller, K. P.; Belcher, A. M.; Schmidt, C. E. Nat. Mater. 2005, 4, 496-502

(48) Yoo, P. J.; Nam, K. T.; Qi, J.; Lee, S. K.; Park, J.; Belcher, A. M.; Hammond, P. T. Nat. Mater. 2006, 5, 234-240.

(49) Menke, E. J.; Thompson, M. A.; Xiang, C.; Yang, L. C.; Penner, R. M. Nat. Mater. 2006, 5, 914-919.

(50) Xiang, C.; Kung, S. C.; Taggart, D. K.; Yang, F.; Thompson, M. A.; Guell, A. G.; Yang, Y.; Penner, R. M. ACS Nano 2008, 2, 1939 1949 .

(51) Lamboy, J. A.; Arter, J. A.; Knopp, K. A.; Der, D.; Overstreet, C. M.; Palermo, E. F.; Urakami, H.; Yu, T. B.; Tezgel, O.; Tew, G. N.; Guan, Z.; Kuroda, K.; Weiss, G. A. I. Am. Chem. Soc. 2009, 131, 16454-16460.

(52) Grant, R. A.; Lin, T. C.; Konigsberg, W.; Webster, R. E. I. Biol. Chem. 1981, 256, 539-546. 